\title{
Artículos
}

\section{Biodiversidad de la fauna malacológica en la laguna de Apoyo, Nicaragua}

\author{
Adolfo López*, Janina Urcuyo y Gabriel Vega
}

Recibido: octubre de 2015 / Aceptado: noviembre de 2015

Debido a su importancia científica y social, en Apoyo se han hecho algunos estudios sobre su valor biológico, entre los que destaca el último hecho por AMICTLAN en 2007 sobre la biodiversidad malacológica, aunque de forma parcial. Los estudios que la UCA ha hecho sobre este particular a lo largo de los años superan los resultados de AMICTLAN y el presente artículo los resume en un listado de 77 especies de moluscos (que incluyen cinco nuevas especies para la ciencia) distribuidas en 25 familias y 42 géneros, y ordenadas en dos tablas. Una de ellas contiene las especies en orden de cantidad de lotes y la otra contiene los mismos lotes pero en orden alfabético de familia, todo ello preservado en la colección del Centro Malacológico de la UCA.

Palabras clave: Laguna de Apoyo / fauna malacológica / biodiversidad

\section{Introducción}

La fauna malacológica terrestre de Nicaragua carece al día de hoy de un estudio sistemático, aunque se ha colectado en muchos lugares y se han publicado listas de las especies registradas, como las de Tate (1869), Fluck (1905-1906) y Jacobson (1965, 1968), quienes solo mencionan un máximo de 70 especies. Desde entonces se han ido añadiendo nuevos registros y descripciones hechas por diversos autores, cuyos artículos

Centro Malacológico de la Universidad Centroamericana, Apdo. 69, Managua, Nicaragua. Correo electrónico: alosi@ns.uca.edu.ni

Centro Malacológico de la Universidad Centroamericana, Apdo. 69, Managua, Nicaragua. Correo electrónico: janina@ns.uca.edu.ni

Centro Malacológico de la Universidad Centroamericana, Apdo. 69, Managua, Nicaragua. Correo electrónico: estacionbiologica@ns.uca.edu.ni 
se encuentran en la literatura desde el año 1968, además de una lista de gastrópodos continentales del Pacífico que contiene aproximadamente 104 especies (Pérez, 1999).

Uno de los programas permanentes del Centro Malacológico de la Universidad Centroamericana (UCACM) es el estudio y conservación de la biodiversidad de fauna y flora de la laguna de Apoyo que forma parte del sistema de lagunas cratéricas del territorio nacional. Este artículo presenta la diversidad de moluscos terrestres y acuícolas en la cuenca de Apoyo, así como comentarios sobre este sistema natural y una lista, resultado del monitoreo efectuado por el personal, estudiantes de ecología y biología asociados al UCACM, con ilustraciones de algunas de las especies para dar a conocer su fauna malacológica endémica.

\section{Metodología}

\section{1. Área de estudio}

La laguna de Apoyo es un lago cratérico de origen volcánico aproximadamente circular, con $20.92 \mathrm{~km}$ de circunferencia, situado en el Sur de Nicaragua entre las ciudades de Masaya y Granada, a unos $40 \mathrm{~km}$ de la costa del Pacífico y posición geográfica UTM 16PFJ0218. Sobre su caldera existió anteriormente uno o varios volcanes que, tras el evento eruptivo más importante registrado en Nicaragua, colapsó hace unos 22.000 años según registros del Instituto Nicaragüense de Estudios Territoriales (INETER, 1967). El nivel de sus aguas está a $75 \mathrm{msnm}$, mientras que el borde superior del cráter llega a los 350 msnm (Vázquez-Prada, Ortega, Alonso \& Cerrato, 2007). Debido en parte a su abrupta pendiente, se forma una unidad ecológica marcadamente singular y aislada.

Sigue habiendo actividad volcánica moderada, que se manifiesta en fumarolas y emanaciones de aguas sulfurosas calientes en algunos puntos del lago. En 1991, el Ministerio del Ambiente y los Recursos Naturales declaró a Laguna de Apoyo como Reserva Natural (Decreto Ejecutivo 44-91, 1991); el área protegida tiene una extensión territorial de 3,500 Ha y mediante este decreto es parte integrante del Sistema Nacional de Áreas Protegidas de Nicaragua (Apoyonic wordpress, 2014). Su área boscosa se clasifica, según zona de vida de Holdridge (1996), como bosque seco tropical. Aproximadamente una cuarta parte de la orilla de la laguna $(7 \mathrm{~km})$ está habitada, aunque la población permanente local no pasa de unas 100 familias (Vianica, 2013). La introducción de plantas y árboles exóticos en el área habitada ha incrementado la biodiversidad de especies en relación con el área despoblada.

\subsection{Materiales y métodos}

La búsqueda de moluscos por parte del UCACM en el área de estudio se comenzó en el año 1988 y la última recolección que se incluye en este informe es del 28 de enero de 2013. Los monitoreos de especies acuícolas se realizaron mediante una serie de incursiones de dos colectores, desde la orilla hasta los $25 \mathrm{~m}$ de profundidad, mediante buceo con equipos SCUBA; el material malacológico se colectó en substratos arenosos del fondo y recogiendo algas de Chara sp. que luego se depositaron en bolsas zip-lock para su posterior revisión y separación de los moluscos. En tierra 
se muestreó desde la orilla misma del agua hasta el borde superior de la caldera, y también en pequeños arroyos que nacen de fuentes en las laderas. Se prestó especial atención a lugares sombreados y frescos bajo árboles, en su mayoría de la familia Fabácea del bosque seco tropical. Igualmente se hicieron revisiones minuciosas debajo de rocas, entre la hojarasca, la tierra y arenisca volcánica a veces calcinada por incendios provocados para abonar el terreno. También se buscaron gastrópodos arbóreos sobre las cortezas y hojas, y en las oquedades de los árboles, raíces y gambas.

Posteriormente, cada lote fue examinado en el laboratorio con un estereoscopio American Optical Corp., modelo 40, con aumentos de 15x y 30x, para separar y determinar las especies presentes con ayuda de manuales de la biblioteca del UCACM (Tate, 1869; Fischer \& Crosse, 1878; Pilsbry, 1894; Martens, 1898 y 1890/1901; Baker, 1930 y 1939; Schalie, 1948; Wenz \& Zilch, 1959 y 1960; Burch, 1962 y 1989; Jacobson, 1965 y 1968; Breure, 1979). Algunos especímenes se lograron determinar con ayuda de especialistas en los museos de Estados Unidos: National Museum, Smithsonian Institution, Washington; Los Angeles County Museum of Natural History, California; State Museum, University of Florida, Gainsville, Florida y Iowa State University. Por intercambio de especímenes, estos museos han empleado material recogido por el Centro Malacológico en estas localidades para sus propias investigaciones.

A cada lote se le asignó una etiqueta señalando el año y el número de la recolección correspondiente y los particulares del lote tal como: Información taxonómica de la especie, referencias bibliográficas, características de localidad, hábitat, fecha, colector, el número de ejemplares y sus dimensiones. También se añadieron detalles particulares que podrían ser de interés ecológico como variaciones morfológicas, anatómicas y de comportamiento. Esta información se contiene en bases Access del UCACM donde se pueden consultar.

\section{Resultados}

Se recolectaron 14,113 especímenes en 355 lotes, con un total de 77 especies de moluscos de 25 familias y 42 géneros (Tablas 1 y 2). La Tabla 1 contiene las especies en orden de cantidad de lotes, empezando por un solo lote para 17 especies, hasta los 57 lotes de Beckianum beckianum. La Tabla 2 contiene los mismos lotes pero en orden alfabético de familia, todo ello preservado en la colección UCACM.

En algunas secciones de la caldera de la laguna las laderas han quedado sin cubierta arbórea debido al despale efectuado para cultivos de subsistencia y para conseguir leña. En tales áreas la población de moluscos es escasa y se halla en marcado contraste con aquellas secciones sombreadas por grandes árboles, algunas con una biodiversidad tan rica como únicamente se halla en la pluviselva de Matagalpa y Jinotega a unos 1,000 msnm: 75 especies para la finca Santa Maura ubicada en la Reserva Natural Datanlí-El Diablo, 43 para Fuente Pura y 26 para Selva Negra (López \& Urcuyo, 2009).

El primer grupo de 17 lotes de un solo ejemplar (Tabla 1), se compone de las especies más raras o más escasas de la laguna. En este grupo se incluyen cinco que se consideran nuevas para la ciencia. 
1. Drymaeus sp. (Ilustración 1), de arrollamiento sinistral, lo que añade uno más a los otros dos Drymaeus sinistrales conocidos de Mesoamérica.

2. Rotadiscus sp. (Ilustración 2), especie de unos 3-4 mm de diámetro, y periostraco hirsuto.

3. Radiodiscus sp. (Ilustración 3), especie variegada de $2.4 \mathrm{~mm}$ de diámetro.

4. Glyphyalinia sp. (Ilustración 4) concha discoidal translúcida, $4 \mathrm{~mm}$ de diámetro, muy común en la geografía baja del país.

5. Systrophia sp. (Ilustración 5) de 2 - 3 mm de diámetro, localmente común por el margen occidental de la laguna.

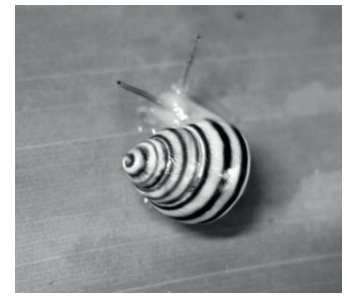

Ilustración 1

Drymaeus sp.

$12 \mathrm{~mm}$

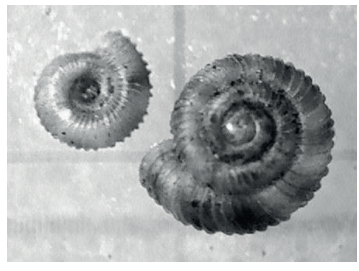

Ilustración 2

Rotadiscus sp. 3-4 mm

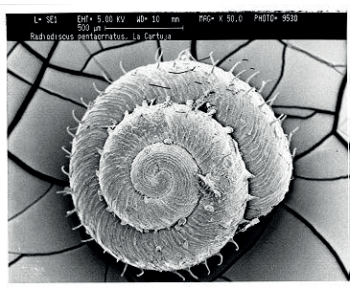

Ilustración 3

Radiodiscus sp. $2.4 \mathrm{~mm}$

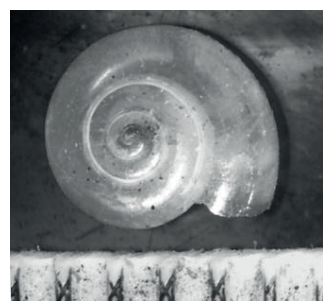

Ilustración 4

Glyphialinia sp.

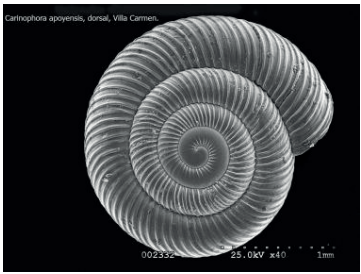

Ilustración $\mathbf{5}$

Systrophia sp.

$2.5 \mathrm{~mm}$

La especie más numerosa fue Beckianum beckianum con 57 lotes mientras que B. sinistrum -su imagen espejo de arrollamiento sinistral- obtuvo 39 lotes; y las especies Lamellaxis gracilis y L. micra alcanzaron 51 y 45 lotes respectivamente. Todas estas especies pertenecen a la familia Subulinidae.

En la Cooperativa J. Quesada al pie de un árbol, en poco más de $1 \mathrm{~m}^{2}$ de tierra y grava volcánica, se recogió una biodiversidad realmente extraordinaria: 736 especímenes de los cuales 650 son Lamellaxis micra, mientras que los restantes 86 forman un lote de 21 especies distintas.

\section{Discusión y comentarios}

Solem y Climo (1985) afirman que las pequeñas comunidades de moluscos se componen de 5 a 12 especies. Ateniéndose a esta cifra, la comunidad malacológica de Apoyo con 77 especies debe considerarse extraordinaria para un área reducida. Previamente sólo habían sido reportadas 38, cuatro de ellas consideradas endémicas (Vázquez, et al. 2007). 
Tanto en cantidad de ejemplares como en diversidad de especies, (biodiversidad [ y biodiversidad () los resultados han sido excepcionales y señalan al ecosistema de esta laguna como uno de los lugares de mayor biodiversidad y productividad malacológica en Nicaragua. Ésta conclusión se ve respaldada por otras investigaciones en ornitología, malacología, botánica (Pérez et al, 2005), que confirman que Apoyo tiene una fauna y flora endémicas que hay que preservar a toda costa por ser únicas.

Las cinco especies indeterminadas consideradas nuevas para la ciencia han sido colectadas en otras localidades de Nicaragua, no solo en Apoyo, por lo que no son endémicas de la Laguna. La primera es un Drymaeus sp. (Ilustración 1) de arrollamiento sinistro, relativamente común en Jinotega y Matagalpa del cual se colectaron dos fragmentos y es evidentemente distinto de D. tropicalis (Morelet, 1849) y D. inusitatus (Fulton, 1900) los otros dos Drymaeus sinistros de Centroamérica. Las siguientes son Rotadiscus sp. (Ilustración 2) de la cual se colectó un único ejemplar y Radiodiscus sp. (Ilustración 3) de escultura hirsuta. Glyphyalinia sp. (Ilustración 4) es abundante (38 lotes) y ubicua en el resto del país. Por último, Systrophia sp., un caracol terrestre helicoidal de uno a tres $\mathrm{mm}$ de diámetro (Ilustración 5), tentativamente de la familia Systrophiidae, abundante (50 lotes) y siempre situado en áreas cercanas a la orilla oeste de la laguna. Fuera de Apoyo solamente se han encontrado cuatro ejemplares en tres puntos aislados, también a orillas de sistemas acuáticos.

Los moluscos acuáticos hallados incluyen cuatro gastrópodos: Pachychilus largillierti (Ilustración 6) se encuentra en el agua de los arroyos que bajan por las laderas, pero no dentro de la laguna. Pyrgophorus coronatus (Ilustración 7) y Aplexa nicaraguana (Ilustración 8), habitan sobre la planta acuática Chara sp. y en la arena. Otro gastrópodo acuático, Melanoides tuberculata, a pesar de ser una especie extremadamente ubicua, queda extinta, en contraste con otros lugares cercanos a Apoyo, donde obtiene una densidad muy grande. La evidencia de su presencia en la laguna es un único lote de dos ejemplares de unos $3 \mathrm{~mm}$ de longitud, la mera protoconcha de esta especie común. Lo extraordinario de este hallazgo es que se hizo en una muestra de tierra recogida por lo menos $30 \mathrm{~m}$ por encima del nivel actual del lago.

Sabemos por comentarios de los habitantes más ancianos del lugar que el agua alcanzaba ese nivel hace unos 100 años y Lorenzo López, maestro de la escuela local y biólogo aficionado (Comunicación personal, 06 de diciembre de 2003) asegura que su abuela, quien murió el año anterior a los 90 años, le decía que cuando era niña, el agua cubría lo que ahora es la carretera. No hemos encontrado evidencia posterior de este caracol en la laguna, aunque es tan abundante en otros lagos y ríos de Nicaragua, especialmente en el cercano Lago Cocibolca, por lo que se deduce que se ha extinguido en Apoyo. La conclusión lógica es que a pesar de una tasa de reproducción muy elevada no se pudo adaptar a la alcalinidad tan alta del agua de Apoyo (Vásquez-Prada et alii, 2007). Algo parecido ocurrió en la laguna de Xiloá con una especie de molusco de la familia Unionidae (Villa, 1982), cuya orilla está cubierta de ejemplares semifósiles. 


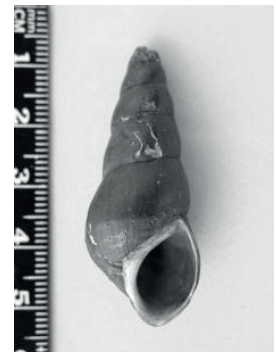

Ilustración 6

Pachychilus largillierti

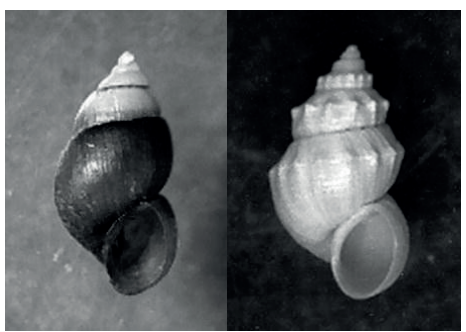

Ilus tración 7

Pyrgophorus coronatus (Liso y coronad o) $3-4 \mathrm{~mm}$

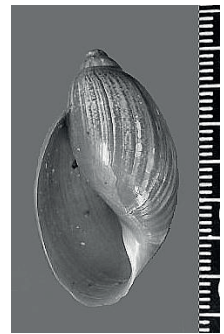

Ilustración 8

Aplexa nicaraguana

En cambio P. coronatus (Ilustración 7) bien conocido por habitar aguas muy contaminadas, ha sabido adaptarse a los cambios de calidad de agua, y tanto en Apoyo como en Xiloá es tan abundante que sus conchas vacías blanquean la arena volcánica gris del substrato. Se halla además en abundancia por las laderas de la caldera de Apoyo en estado semifósil, dando evidencia de la caída drástica del nivel freático que se tiene por $0.30 \mathrm{~m} /$ año (Vázquez-Prada et al., 2007). Una característica de este caracol es la gran variedad de su escultura y ornamentación, siendo algunos ejemplares totalmente lisos mientras que otros desarrollan estrías, cóstulas o coronaciones fuertemente marcadas dando lugar a confusión entre autores y como consecuencia a 6 taxones para esta especie. Otras especies del mismo género también muestran estas variaciones (Thompson, 1968), lo que ha suscitado discusiones acerca de su origen, entre otros atribuido a la alcalinidad del agua o a presencia algal (Schalie, 1948). En Apoyo el agua es fuertemente alcalina, pero la mayoría de ejemplares son lisos, lo que parece negar que sea esta calidad la que provoca escultura nodulosa. Otra razón que ha sido aducida (McKaye et al., 1992) es que los caracoles desarrollan esta escultura como defensa contra depredadores, en este caso, de peces de la familia Cichlidae que se alimentan de ellos. Lo que implica posibilidades investigativas para la verificación de variedades específicas de los peces por la presencia de caracoles en su contenido estomacal y la correlación con los dientes faríngeos que les permiten triturarlos.

Entre los caracoles terrestres, la familia SUBULINIDAE es una de las mejor representadas con cinco géneros y 11especies. Belt (1928) reportó para esta familia un ejemplar juvenil de $4 \mathrm{~mm}$ de longitud, hallado en Acoyapa y descrito anteriormente por Martens (1898) como Leptinaria sinistra endémica de Nicaragua. La especie es sinistral (Ilustración 9) y en Apoyo se han recogido más de 200 ejemplares en 39 lotes, juveniles y adultos, hasta una longitud de $10 \mathrm{~mm}$. Beckianum beckianum (Ilustración 10), especie dextral gemela, es todavía más abundante (Pfeiffer, 1846), y en todo parecida. Algunas colonias constan de una de las dos especies solamente, como en la laguna de Asososca, León, donde únicamente se halla la sinistral y en abundancia, mientras que en otras localidades solamente la dextral y todavía en otras las dos. Esto sugiere la identidad específica de las dos especies: B. beckianum y B. sinistrum (Pérez \& López, 1995).

Otro hallazgo notable, Spiraxis alvaradoi (Ilustración 11) y S. funibus cuya microescultura es casi igual, a pesar de la diferencia de tamaño, perfil y protoconchas, y son generalmente compátridas en varias localidades del país. 
Encuentro №. 102, 8-18, 2015

La presencia de Biomphalaria glabrata (Ilustración 12) es causa de alarma por ser potencial portadora de la cercaria Schistosomiasis mansoni, la enfermedad del sueño en el área de América Central y Caribe. Sin embargo, no se ha reportado la enfermedad en Nicaragua. Malek (1962), reconoce que existe ausencia de infección de ciertos tremátodos en algunas áreas donde se da la especie de caracol que suele ser su hospedero.

Euglandina cumingi (Ilustración 13a) se encuentra presente en todo el territorio nacional hasta alturas superiores a los $1000 \mathrm{msnm}$. Se les conoce como "caracoles lobo" por sus características de ágiles depredadores, alimentándose de otros caracoles y babosas que son plagas en diversos cultivos de importancia nacional. Las Euglandinas exhiben una rádula (Ilustración 13b) provista de un sinnúmero de dientes con los que despedazan sus presas, dejando sus conchas totalmente limpias.

La laguna de Apoyo (Ilustración 14), además de ser un ecosistema digno de estudio científico, es un lugar turístico por su belleza natural.
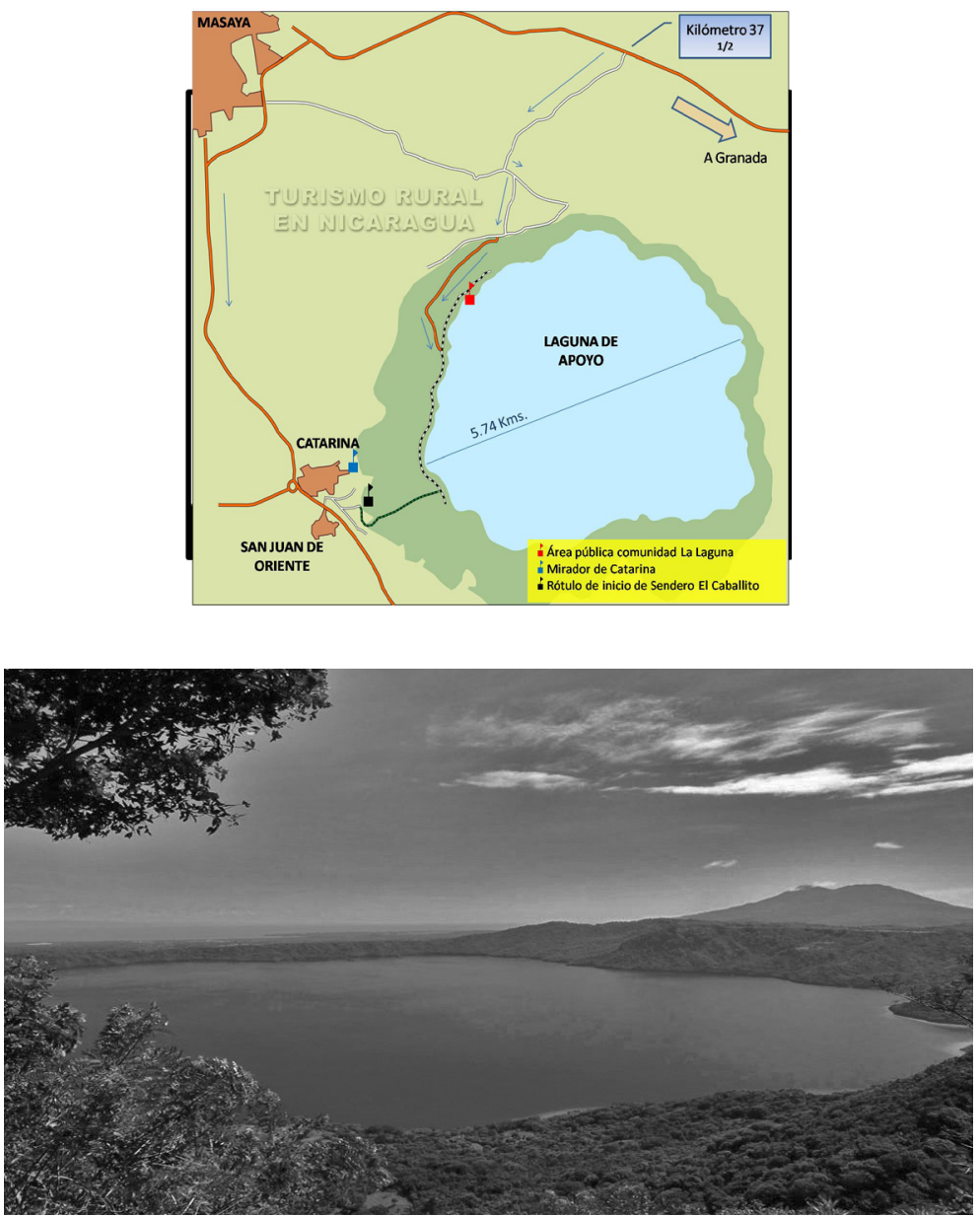

Ilustración 14. Panorámica (WTNTRAVEL, 2015) y mapa de Laguna de Apoyo (Centeno, 2011) 
Tabla 1. Lista de especies de moluscos, laguna de Apoyo, por cantidad de lotes

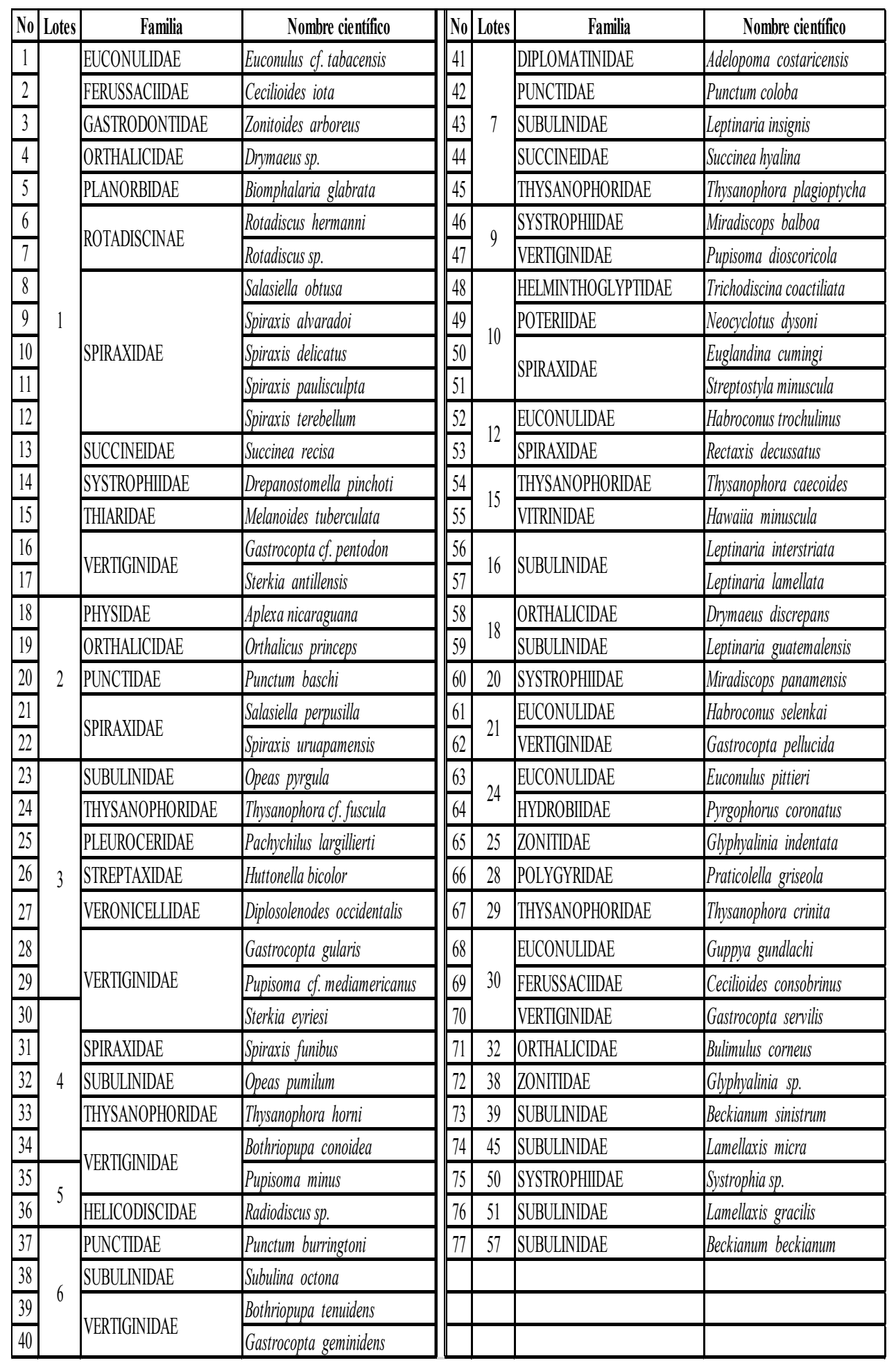


Encuentro №. 102, 8-18, 2015

Tabla 2. Lista de especies de moluscos, laguna de Apoyo, en orden alfabético de familias

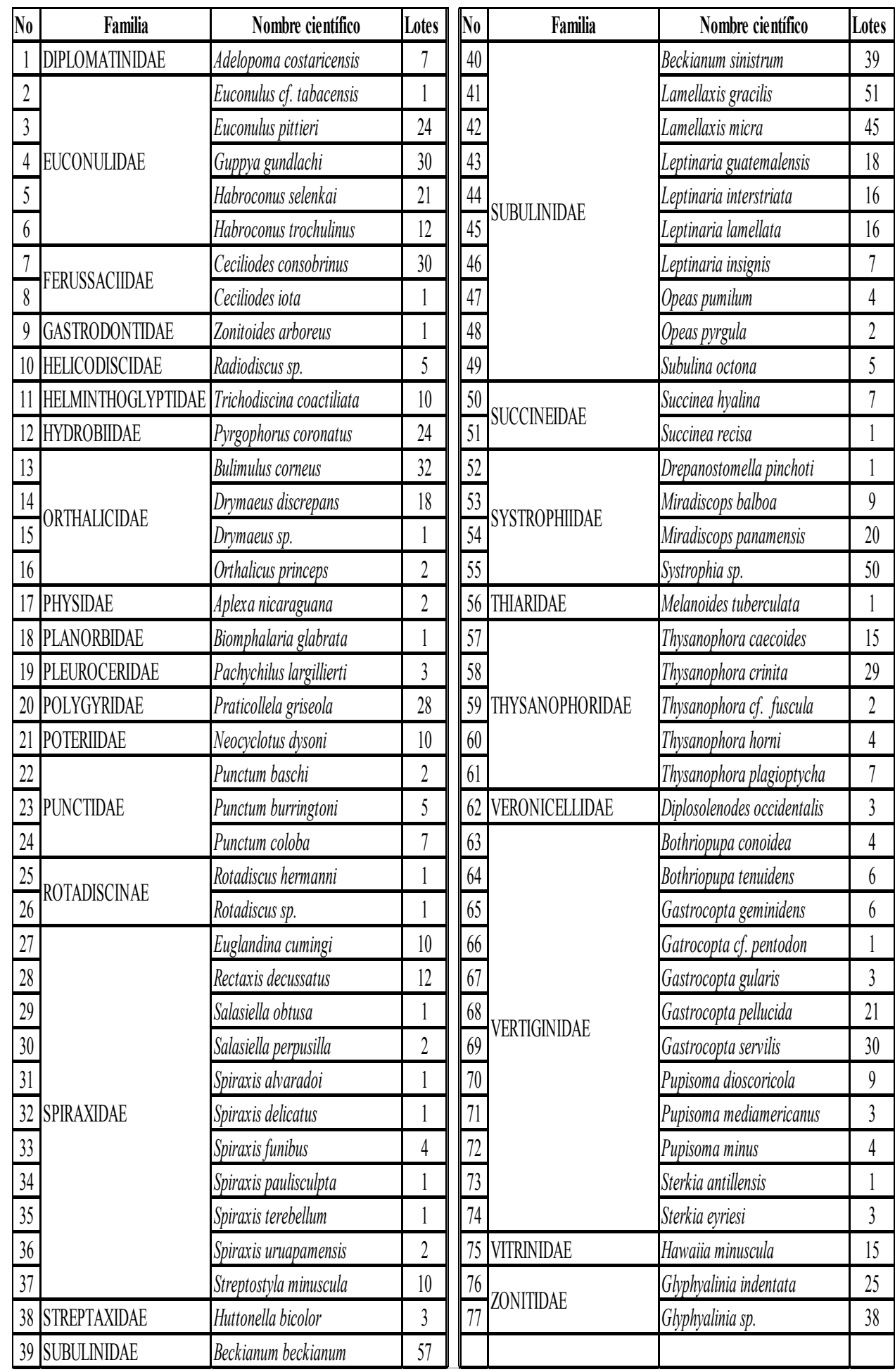




\section{Referencias bibliográficas}

Apoyonic wordpress. (2014). Laguna de Apoyo. Recuperado de http:// lagunadeapoyonic. wordpress.com/reserva-natural/

Baker, H. B. (1930). Mexican mollusks collected for Dr. Bryant Walker in 1926. Occ. Pap. of Mus. of Zool., U. of Michigan, (220), 22-24.

Baker, H. B. (1939). A revision of Spiraxis C.B. Adams. Nautilus, 53(1), 8-16.

Belt, T. (1911). The Naturalist in Nicaragua. Temple Press, London.

Breure, A. S. H. (1979). Systematics, Phylogeny and Zoogeography of Bulimulinae (Mollusca). Leiden: Zoologische Verhandelingen.

Burch, J. B. (1962). How to know the eastern land snails. Dubuque, Iowa: W. M. Brown Co. Publ.

Burch, J. B. (1989). North American Freshwater Snails. Michigan: Malacological Publications.

Fischer, P. \& Crosse, H. (1878). Mission Scientifique au Mexique et dans L' Amerique Centrale. Mollusques Terrestres et Fluviatiles. Paris: Imprimerie Nationale, (1), $1-702$.

Fluck, W. H. (1905). Shell collecting on the Mosquito Coast of Nicaragua. Nautilus, I-V, (19), 8-12, 16-19, 32-34, 55-57, 78-80.

Fluck, W. H. (1906). Shell collecting on the Mosquito Coast of Nicaragua. Nautilus, VI, (20),1-4.

Fulton, H. C. (1900). Bulimulus (Drymaeus) inusitatus. Nautilus, (16), 87.

Holdridge, L. R. (1996). Ecología basada en zonas de vida (5ta. Ed.). San José, C.R.: Instituto Interamericano de Cooperación para la Agricultura.

Instituto Nicaragüense de Estudios Territoriales. (1967). Mapa Básico de Nicaragua. Managua.

Jacobson, M. K. (1965). Preliminary remarks on the land mollusks of Nicaragua. Annual Reports of the American Malacological Union, (3).

Jacobson, M. K. (1968). On a collection of terrestrial mollusks from Nicaragua. Nautilus, 81, (4), 114-120.

López A. \& Urcuyo, J. (2009). Moluscos de Nicaragua II: Gastrópodos (1ra. ed.). Managua: MARENA.

Malek, E. A. (1962). Laboratory Guide and Notes for Medical Malacology (15a ed.). Minneapolis: Burgess.

Martens, E. von. (1890-1901). Biologia Centrali-Americana. Land and Freshwater Mollusca. London: Zoological Society of London.

Martens, E. von. (1898). Mollusca. Malacological Review. (28),127-130.

McKaye, K., Ryan, J., Stauffer, J., López, L., Vega, G., \& van den Berghe, E. (1992). African Tilapia in Lake Nicaragua. Ecosystem in transition. Oxford: BioScience.45, (6).

Ministerio del Ambiente y Recursos Naturales (MARENA). (1991, 31 de octubre). Decreto Ejecutivo 44-91. Declaración de Áreas Protegidas en varios Cerros, Macizos montañosos, Volcanes y Lagunas del país. Publicada en La Gaceta Diario Oficial, (207). 
Encuentro №. 102, 8-18, 2015

Morelet, A. (1849). Testacea novissima insulae Cubanae et Americae Centralis. Paris: I. J.-B. Baillière.

Pérez, M. (1999). Estudio Taxonómico y Biogeográfico Preliminar de la Malacofauna Continental (MOLLUSCA: GASTROPODA) del Pacífico de Nicaragua (Tesis inédita de Doctorado). Universidad del País Vasco, Leioa, España.

Pérez, M. \& López, A. (1995). Rediscovery, distribution and taxonomic assignment of Leptinaria sinistra. Malacological Review. 28 (1/2),127-130.

Pérez, M., Sotelo, M., Ramírez, F., Ramírez, I., López, A. \& Sirias, I. (2005). Composición y Diversidad de las comunidades de aves, moluscos y plantas asociadas con sistemas silvopastoriles de Matiguas y Río Blanco, Dpto. de Matagalpa, Nicaragua. Encuentro, (71), 136-165.

Pilsbry, H.A. (1894): Manual of Conchology. Philadelphia: Published by the Author.

Schalie, H. v.d. (1948). The land and freshwater mollusks of Puerto Rico. Miscellaneous. Publications, U. of Mich., (70), 134.

Solem, A. \& Climo F. M. (1985). Structure and habitat correlations of Sympatric New Zealand snail species. Malacología 26, (1-2),1-30.

Tate, R. (1869). On the land and fresh-water mollusca of Nicaragua. Am. Jr. of Conch, (5),151-162.

Thompson, F. G. (1968). The Aquatic Snails of the Family HYDROBIIDAE of Peninsular Florida. Gainsville: U. of Florida Press.

Vázquez-Prada, D., Ortega, J., Alonso, E., Cerrato, D. (2007). Estudio Hidrogeológico y Gestión de los Recursos Hídricos de la Cuenca de la Laguna de Apoyo. (AMICTLANManagua). Recuperado de http://es.scribd.com/doc/38156038/ Hidrogeologico-Laguna-de-Apoyo\#scribd

Wenz, W. \& Zilch, A. (1959/1960). Gastropoda. Euthyneura. En Handbuch der Palaeozoologie. Berlin: Band 6, Teil 2, Liefrng. 1-4; 834 pp.

Vianica. (2013). Laguna de Apoyo. Masaya. Recuperado de: http://vianica.com/sp/go/ specials/25-laguna-de-apoyo-nicaragua.html.

Villa, J. (1982). Peces nicaragüenses de agua dulce. Managua: Ed. Fondo de Promoción Cultural Banco de América.

WTNTRAVEL. (2015). Recuperado de http://www.welcometonicaragua.net/es/ package/apoyo-lagoon-granada-islets/ 